\title{
Stepwise phenological adaptation
}

Nat. Genet. http://doi.org/dqx8 (2020).
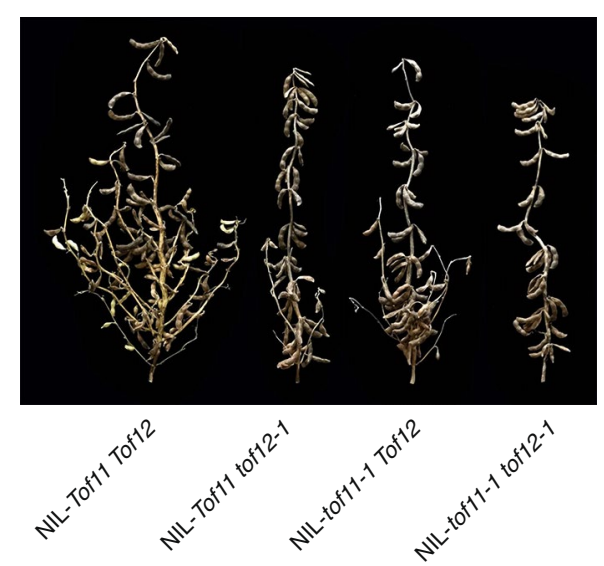

Credit: Image courtesy of Fanjiang Kong

Initially domesticated in the temperate regions of China, cultivated soybean expanded its geographical distribution toward the north by shifting to an early flowering and maturity habit. Although a number of genetic variants have been found to account for the variations of flowering time in soybean, the genes facilitating this change for early latitudinal adaptation remains unknown. Sijia Lu, from Guangzhou University, and colleagues now report two homologous pseudo-response regulator $(P R R)$ genes, Tof12 and Tof11, that sequentially contributed to high-latitude adaptation during early soybean domestication.

Using a panel consisting of 424 newly resequenced accessions and another panel of 809 previously re-sequenced accessions, as well as a bi-parental population, the researchers detected loci associated with flowering times by association and linkage mapping. Two loci located in chromosomes 11 and 12, referred to as Tof 11 and Tof 12 , were detected in all three populations and further narrowed down to two $P R R$ genes. Their roles in controlling flowering time were validated by transgenic complementation.
Genetic analyses support that Tof 11 and Tof 12 function independently with some redundancy, and their function depends on the legume-specific E1 gene. E1 represses two key FLOWERING LOCUS $T(F T)$ homologues, FT2 $a$ and FT5 a, and thereby suppresses flowering under long day conditions. E1 gene is repressed by LATE ELONGATED HYPOCOTYL (LHY) genes which can be repressed by Tof 11 and Tof 12 . So Tof 11 and Tof 12 overexpression increases E1 expression and consequently represses the expression of the FT homologues, while the loss-of-function mutations, tof 11 and tof 12, derepress FT2 $a$ and FT5a, leading to early flowering. Figure 1 shows four near-isogenic lines (NILs) with different Tof11/tof 11 and Tof12/tof12 genotypes and their corresponding flowering and maturity phenotypes.

A very strong selection signature was detected around the Tof 12 gene, with a loss-of-function tof 12 mutation strongly favoured in landraces and fixed in all improved cultivars. Loss-of-function tof 11 have arisen multiple times, but only one mutation that occurred in the background of the tof 12 mutation becomes widespread in domesticated soybean. Molecular dating suggests that this tof 11 mutation occurred 2,500 years after the tof 12 mutation, so they were sequentially incorporated into the cultivated soybean gene pool, conferring an additive early flowering phenology.

The tof 12 mutation experienced artificial selection in a strength similar to that experienced by other well-known soybean domestication genes regulating seed dormancy and shattering, suggesting these phenotypes may have been selected in parallel. The allele frequency patterns of the three genes, Tof12, Tof 11 and E1, indicated they were sequentially adopted in breeding for high-latitude adaptation.

\section{Jun Lyu}

Published online: 9 April 2020 https://doi.org/10.1038/s41477-020-0644-0 